\title{
The astrocyte marker Aldh1L1 does not reliably label enteric glial cells
}

\author{
Werend Boesmans ${ }^{\mathrm{a}}$, Natália Pessoa Rocha ${ }^{\mathrm{a}, \mathrm{b}}$, Helton José Reis ${ }^{\mathrm{a}, \mathrm{b}}$, \\ Matthew Holt ${ }^{\mathrm{c}, \mathrm{d}}$, Pieter Vanden Berghe ${ }^{\mathrm{a}, *}$ \\ a Laboratory for Enteric NeuroScience (LENS), Translational Research Center for GastroIntestinal Disorders (TARGID), Department of Clinical and \\ Experimental Medicine, University of Leuven, Leuven, Belgium \\ ${ }^{\mathrm{b}}$ Laboratório de Neurofarmacologia, Instituto de Ciências Biológicas, Universidade Federal de Minas Gerais, Belo Horizonte, Brazil \\ c The Laboratory of Glia Biology, Department of Human Genetics, University of Leuven, Leuven, Belgium \\ ${ }^{\mathrm{d}}$ VIB Center for the Biology of Disease, Leuven, Belgium
}

\section{H I G H L I G H T S}

- The expression of the astrocytic marker Aldh1L1 was studied in the enteric nervous system using Aldh1L1-eGFP BAC transgenic mice.

- Only enteric glial cells outside the ganglia showed Aldh1L1 promoter activity.

- Aldh1L1-eGFP expression was also observed in interstitial cells of Cajal and in a subpopulation of excitatory enteric neurons.

\section{A R T I C L E I N F O}

\section{Article history:}

Received 17 January 2014

Received in revised form 17 February 2014

Accepted 20 February 2014

\section{Keywords:}

Aldh1L1

Enteric glial cell

Enteric neuron

Enteric nervous system

Astrocyte

\begin{abstract}
A B S T R A C T
Enteric glial cells are increasingly acknowledged as important partners of enteric neurons in the control of gastrointestinal function. They share morphological features and expression of antigenic markers with astrocytes of the central nervous system. Recently, aldehyde dehydrogenase 1 family member L1 (Aldh1L1) has been proposed as a novel and specific marker for astrocytes. Taking the known similarities between astrocytes and enteric glia into account, we sought to investigate whether enteric glial cells also express Aldh1L1. To this end, we performed immunostaining on preparations of myenteric plexus obtained from adult Aldh1L1-eGFP bacterial artificial chromosome (BAC) transgenic mice and found that the Aldh1L1 promoter is indeed active in enteric glia, albeit mainly in cells residing outside the myenteric ganglia. Apart from enteric glia, we also observed eGFP expression in interstitial cells of Cajal. Furthermore, myenteric ganglia of the large intestine contained eGFP positive neurons. Taken together, our data indicate that Aldh1L1 is not a suitable marker for enteric glial cells.
\end{abstract}

(c) 2014 Elsevier Ireland Ltd. All rights reserved.

\section{Introduction}

Similar to other parts of the nervous system, the enteric nervous system (ENS) is composed of neurons and glia. This neural network is situated within the gut wall and autonomically controls many aspects of gastrointestinal function independent of the brain [1]. Over the last two decades, our knowledge of enteric glial cell function has evolved from thinking of them acting merely in a simple neuro-supportive role to encompass an intriguingly diverse range of roles in maintaining gut homeostasis. Enteric glial cells are involved in mucosal barrier protection, inflammatory reactions and motility [2-4]. Enteric glia are phenotypically similar to central

\footnotetext{
* Corresponding author at: Laboratory for Enteric NeuroScience, TARGID, University of Leuven, Herestraat 49, ON1, mailstop 701, B-3000 Leuven, Belgium. Tel.: +32 16330153 .

E-mail address: pieter.vandenberghe@med.kuleuven.be (P. Vanden Berghe).
}

nervous system (CNS) astrocytes; in addition to sharing morphological features, enteric glial cells and astrocytes both express the intermediate filament glial fibrillary acidic protein (GFAP) and the $\mathrm{Ca}^{2+}$ binding protein $\mathrm{S} 100 \beta$ [5]. Recently, transcriptional profiling studies of astroglia in the CNS have identified aldehyde dehydrogenase 1 family member L1 (Aldh1L1) as a new marker for astrocytes and astrocytic precursors [6-8]. Aldh1L1, also named 10formyltetrahydrofolate dehydrogenase ( $\mathrm{FDH})$, is a folate enzyme that converts 10-formyltetrahydrofolate to tetrahydrofolate [9]. It is crucial in several biochemical reactions, including de novo nucleotide biosynthesis and regeneration of methionine, rendering it of great influence in cell division and growth. Using Aldh1L1 as an astrocyte marker was originally proposed based on data arguing that Aldh1L1 is highly, broadly and specifically expressed by nearly all astrocytes, but not other CNS cell types [6,8]. Given the many similarities between enteric glial cells and astrocytes, the aim 
of the current study was to investigate whether Aldh1L1 would also be a suitable marker for the glial cells in the ENS.

\section{Methods}

Adult Aldh1L1-eGFP bacterial artificial chromosome (BAC) transgenic mice (GENSAT project [10]) of either sex were used. These animals express an eGFP reporter gene under control of the Aldh1L1 promoter, which is thought to accurately reflect Aldh1L1 protein expression [6-8]. Animals were killed by cervical dislocation, under approval by the Animal Ethics Committee of the University of Leuven. The gut was isolated, cut along the mesenteric border and pinned flat in a Sylgard Petri dish containing Krebs solution continuously oxygenated with carbogen $\left(95 \% \mathrm{O}_{2} / 5 \%\right.$ $\mathrm{CO}_{2}$ ). The mucosal and submucosal layers were peeled away and the tissue was fixed in $4 \%$ paraformaldehyde-containing Krebsbuffered solution for $45 \mathrm{~min}$ at room temperature. After washing in PBS, the circular muscle layer was carefully removed to expose the myenteric plexus and the tissues were then processed for immunohistochemistry. The myenteric plexus preparations were permeabilized with $0.5 \%$ Triton X-100 in PBS containing $4 \%$ donkey serum and incubated in the following primary antibodies for $24 \mathrm{~h}$ at $4{ }^{\circ} \mathrm{C}$ : rat anti-GFP (which also detects eGFP; 1:1000; Gentaur, Kampenhout, Belgium); goat anti-Sox10 (1:300; Santa Cruz Biotechnologies, Santa Cruz, CA, USA); rabbit anti-S100 $\beta$ (1:500; Dako, Glostrup, Germany); mouse anti-HuCD (1:500; Molecular Probes, Invitrogen, Merelbeke, Belgium); goat anti-c-Kit (1:200; Santa Cruz Biotechnologies); rabbit anti-nNOS (1:400; Santa Cruz Biotechnologies); rabbit anti-calretinin (1:2000; Chemicon International, Temecula, CA, USA); rabbit anti-calbindin D-28K (1:1600; Swant, Bellinzona, Switzerland) and rabbit anti-neurofilament $\mathrm{M}$ $145 \mathrm{kDa}$ (1:1000; Chemicon International). After washing, secondary antibodies raised in donkey were applied for $2 \mathrm{~h}$ at room temperature: anti-rat Alexa 488 (1:1000; Molecular Probes, Invitrogen); anti-goat Alexa 594 (1:1000; Molecular Probes, Invitrogen); anti-rabbit AMCA (1:250; Jackson Laboratories, West Grove, PA, USA) and anti-rabbit Alexa 594 (1:1000; Molecular Probes, Invitrogen). Preparations were imaged using a Zeiss
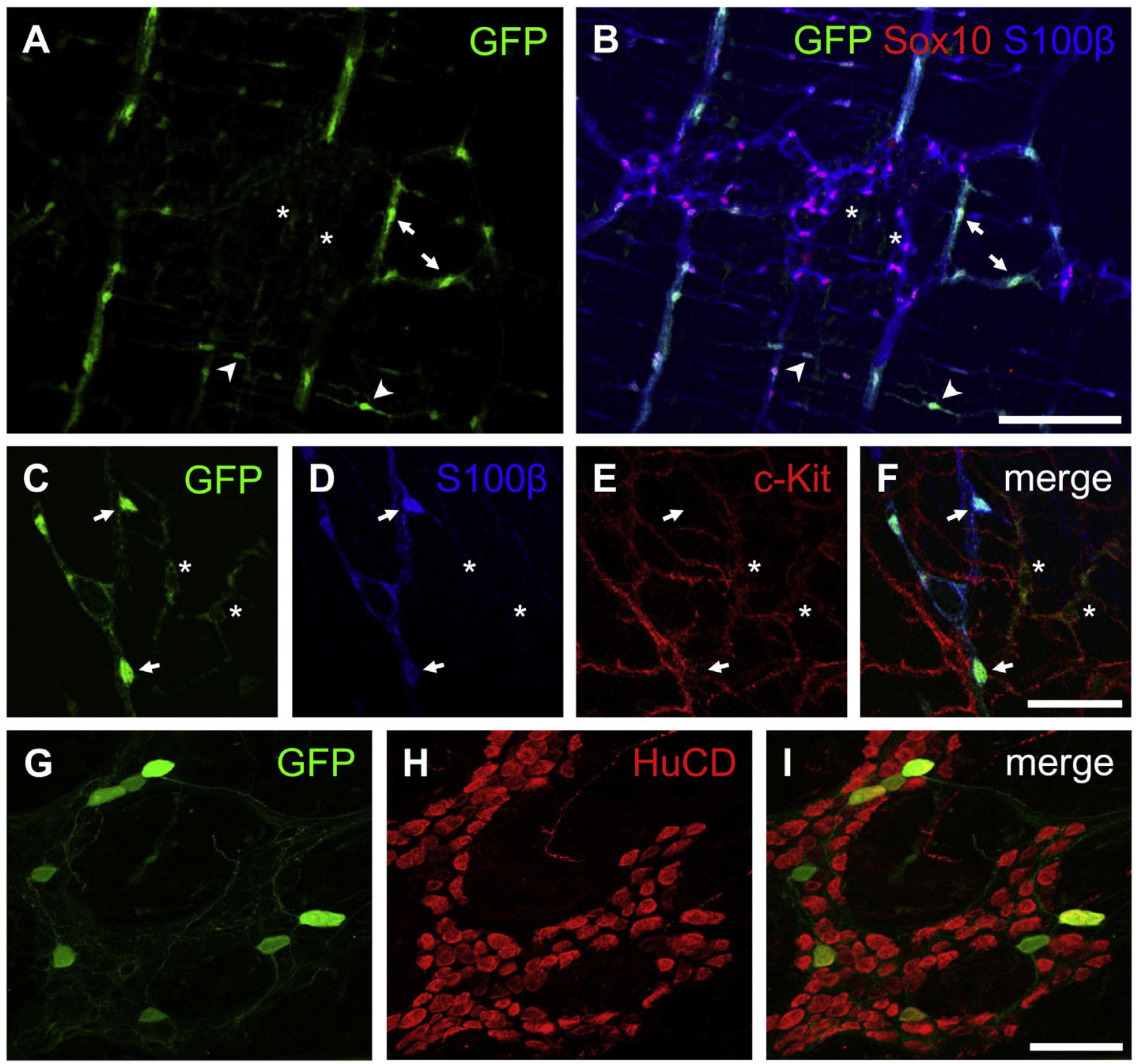

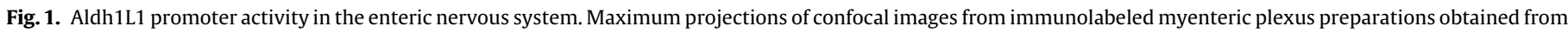

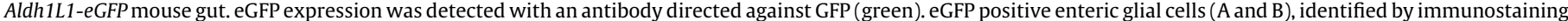

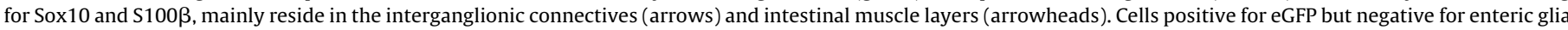

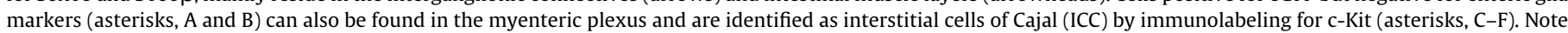

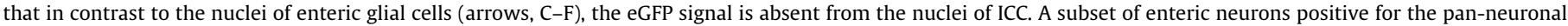
marker HuCD (G-I) expresses eGFP under control of the Aldh1L1 promoter.

Scale bars $=100 \mu \mathrm{m}$ in (A-B and G-I); $50 \mu \mathrm{m}$ in (C-F). 
LSM510 multiphoton confocal microscope (Cell Imaging Core, University of Leuven). All observations and associated statistics were obtained from specimens derived from at least three mice. Values are given as mean \pm standard error of the mean (SEM). The $n$ value refers to the total number of eGFP positive cells counted.

\section{Results}

To investigate the expression of Aldh1L1 in the mouse ENS, we performed anti-GFP immunostaining on preparations of myenteric plexus obtained from Aldh1L1-eGFP mouse gut. eGFP immunoreactivity was detected in enteric glial cells and confirmed by co-labeling with antibodies against the enteric glia markers $S 100 \beta$ and Sox10 (Fig. 1A and B). Interestingly, the majority of eGFP positive enteric glial cells were located outside the myenteric ganglia, i.e. in the interganglionic connectives, tertiary plexus and muscle layers. In the myenteric plexus preparations from the small intestine, we also observed reporter expression in interstitial cells of Cajal (ICC) in addition to enteric glial cells, as confirmed by double labeling for GFP and c-Kit (Fig. 1C-F). Surprisingly, we found that myenteric ganglia of the distal gut contained neurons positive for eGFP, as identified by co-labeling for the pan-neuronal marker HuCD (Fig. 1G-I). A quantification of the different cell types expressing eGFP in the myenteric plexus of the ileum is shown in Table 1 . In contrast with the small intestine where eGFP positive neurons are very rare (Table 1 ), approximately one third $(32.0 \pm 3.2 \%)$ of the colonic ganglia are populated with eGFP positive neurons. These neurons had large and smooth cell bodies, resembling neuronal type II morphology. Because of the consistent and specific expression pattern of the eGFP positive neurons, we performed a set of double stainings to further investigate the neurochemical coding of these neurons and possibly pinpoint a specific subtype of enteric neurons. $77.1 \pm 3.6 \%$
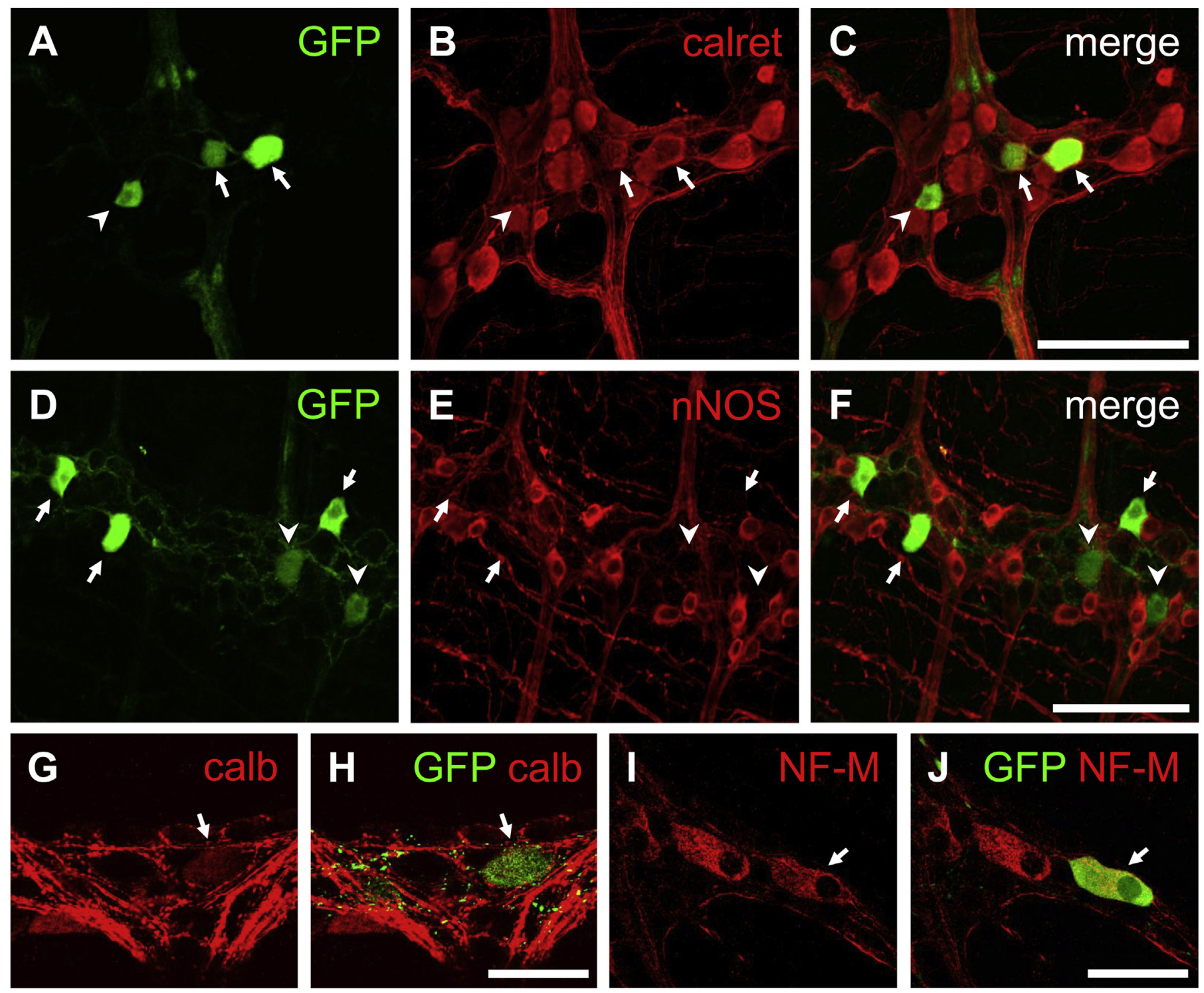

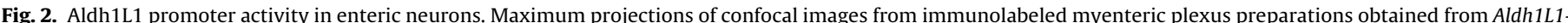

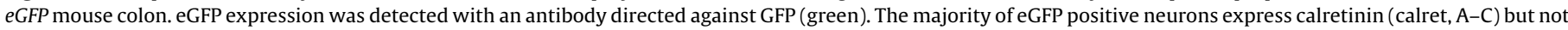

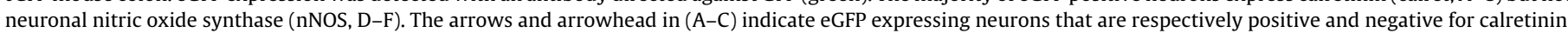

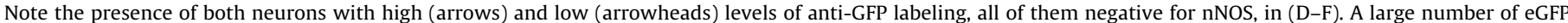
neurons are positive for calbindin (calb, arrow, G and H) and neurofilament-M $145 \mathrm{kDa}$ (NF-M, arrow, I and J).

Scale bars $=100 \mu \mathrm{m}$ in (A-C and D-F); $50 \mu \mathrm{m}$ in ( $\mathrm{G}$ and $\mathrm{H}$; I and $\mathrm{J}$ ). 
Table 1

Quantification of AldhL1-eGFP expressing cells in the myenteric plexus.

\begin{tabular}{lcl}
\hline Cell type & $\begin{array}{l}\text { \% of eGFP cells } \\
( \pm \text { SEM })\end{array}$ & $\begin{array}{l}\text { Number of cells } \\
\text { counted }\end{array}$ \\
\hline Enteric glial cells & $70.0 \pm 4.7$ & 836 \\
Ganglionic glia & $12.5 \pm 1.4$ & 149 \\
Extraganglionic glia & $57.5 \pm 3.5$ & 687 \\
ICC & $28.8 \pm 4.3$ & 310 \\
Enteric neurons & $1.1 \pm 0.4$ & 11 \\
\hline
\end{tabular}

( $n=175$ ) of the eGFP positive neurons were found to express calretinin but none $(n=151)$ were positive for neuronal nitric oxide synthase (nNOS) (Fig. 2A-F), thus indicating their excitatory nature. Next, we performed double stainings for GFP and calbindin or GFP and neurofilament-M $145 \mathrm{kDa}$ (Fig. 2G-J). A rather faint calbindin immunoreactivity was observed in about half $(50.3 \pm 1.7 \%, n=96)$ of the eGFP positive neurons. In addition, $62.5 \pm 4.2 \%(n=66)$ of the eGFP positive neurons were labeled with an antibody directed to neurofilament-M $145 \mathrm{kDa}$.

\section{Discussion}

In the current study, we used expression of an eGFP reporter gene to demonstrate Aldh1L1 promoter activity (and likely protein expression) in glial cells of the ENS, to see if it mimicked the previously described expression in astroglia of the CNS [6]. Although not all enteric glial cells were found to be positive for eGFP, our data further strengthen the notion that enteric glial cells are similar to astrocytes. Remarkably, eGFP was predominantly expressed by enteric glia residing outside rather than within the myenteric ganglia. Using both transgenic lines and antibodies, almost complete Aldh1L1 labeling of astrocytes has been shown throughout the brain, which justifies its use as a novel astrocytic marker $[7,8]$. Thus, our findings indicate that Aldh1L1 most likely cannot be used as a general marker for glial cells in the gut and we hypothesize that the selective expression of Aldh1L1 might reflect enteric glial cell heterogeneity [11]. Moreover, we also observed reporter expression in ENS cell types other than enteric glia. eGFP was present in a subset of enteric neurons, in particularly in the myenteric plexus of the colon. This needs to be assessed in light of the fact that although Aldh1L1 is generally accepted as a suitable marker for CNS astrocytes, Aldh1L1 transcriptional activity has also been found in some neurons in the anterior dorsal thalamus [12]. In our experiments, the immunoreactivity for calretinin, calbindin and neurofilament-M $145 \mathrm{kDa}$, but not for nNOS, as well as the type II-like morphology, suggests that the enteric neurons expressing the reporter are a subset of intrinsic primary afferent neurons, as described by Qu et al. in the mouse ileum [13]. Future studies will need to address whether these neurons have the electrophysiological and morphological characteristics associated with this type of enteric nerve cell. In addition to enteric glia and neurons, we also found Aldh1L1 promoter activity in ICC. This is remarkable since ICC are developmentally unrelated to enteric neurons and glia; they originate from the mesenchyme, while enteric neurons and glia develop from common neural crest precursors [14,15]. Fate mapping using Aldh1L1-Cre mice would be helpful in understanding the temporal control of Aldh1L1 expression in the ENS [7]. Of course, it is also worth investigating what the specific role of the Aldh1L1 protein, and thus the importance of folate metabolism, is in these gut cells. In conclusion, although Aldh1L1 is increasingly used as an astrocyte marker in the CNS, our data indicate that it does not reliably label enteric glial cells in the gastrointestinal tract.

\section{Funding}

This work was funded by BOF, University of Leuven (OT ZKC1808) and the Fonds voor Wetenschappelijk Onderzoek (FWO, Belgium; KN 1.5.135.06; G.0501.10). Confocal recordings were made on the equipment of the Cell Imaging Core (which is supported by Hercules foundation grants). Werend Boesmans is a postdoctoral fellow of the FWO. Natália Pessoa Rocha is a CNPq SWO scholarship recipient (Brazil). Helton José Reis is a recipient of a CNPq fellowship (Brazil) and supported by FAPEMIG. Matthew Holt is the recipient of an ERC Starting Grant (281961; AstroFunc).

\section{Disclosures}

The authors have no financial interests to disclose.

\section{Author contributions}

Experiments, interpretation of data, drafting and editing of the manuscript were done by NPR and WB. Study concept was designed by WB, HJR and PVB. MH provided Aldh1L1-eGFP mice, aided in interpretation of data and critically revised the manuscript. PVB supervised the study and obtained funding.

\section{Acknowledgements}

We would like to thank the members of LENS for their critical comments and skilled technical assistance, and Jessica Bouhuijzen Wenger for animal husbandry.

\section{References}

[1] J.B. Furness, The Enteric Nervous System, Blackwell Publications, Malden, MA, 2006, p. 274

[2] B.D. Gulbransen, K.A. Sharkey, Novel functional roles for enteric glia in the gastrointestinal tract, Nat. Rev. Gastroenterol. Hepatol. 9 (2012) 625-632.

[3] M. Neunlist, L. Van Landeghem, M.M. Mahe, P. Derkinderen, S.B. des Varannes, M. Rolli-Derkinderen, The digestive neuronal-glial-epithelial unit: a new actor in gut health and disease, Nat. Rev. Gastroenterol. Hepatol. 10 (2013) 90-100.

[4] R. De Giorgio, F. Giancola, E. Boschetti, H. Abdo, B. Lardeux, M. Neunlist, Enteric glia and neuroprotection: basic and clinical aspects, Am. J. Physiol. Gastrointest. Liver Physiol. 303 (2012) G887-G893.

[5] M.D. Gershon, T.P. Rothman, Enteric glia, Glia 4 (1991) 195-204

[6] J.D. Cahoy, B. Emery, A. Kaushal, L.C. Foo, J.L. Zamanian, K.S. Christopherson, Y. Xing, J.L. Lubischer, P.A. Krieg, S.A. Krupenko, W.J. Thompson, B.A. Barres, A transcriptome database for astrocytes, neurons, and oligodendrocytes: a new resource for understanding brain development and function, J. Neurosci. 28 (2008) 264-278

[7] A.C. Tien, H.H. Tsai, A.V. Molofsky, M. McMahon, L.C. Foo, A. Kaul, J.D. Dougherty, N. Heintz, D.H. Gutmann, B.A. Barres, D.H. Rowitch, Regulated temporal-spatial astrocyte precursor cell proliferation involves BRAF signalling in mammalian spinal cord, Development 139 (2012) 2477-2487.

[8] Y. Yang, S. Vidensky, L. Jin, C. Jie, I. Lorenzini, M. Frankl, J.D. Rothstein, Molecular comparison of GLT1+ and ALDH1L1+ astrocytes in vivo in astroglial reporter mice, Glia 59 (2011) 200-207.

[9] S.A. Krupenko, FDH: an aldehyde dehydrogenase fusion enzyme in folate metabolism, Chem. Biol. Interact. 178 (2009) 84-93.

[10] N. Heintz, BAC to the future: the use of bac transgenic mice for neuroscience research, Nat. Rev. Neurosci. 2 (2001) 861-870.

[11] W. Boesmans, P. Van Den Berghe, V. Pachnis, Glial heterogeneity in the enteric nervous system, Neurogastroent. Motil. 24 (Suppl. 2) (2012) 36

[12] L.C. Foo, J.D. Dougherty, Aldh1L1 is expressed by postnatal neural stem cells in vivo, Glia 61 (2013) 1533-1541.

[13] Z.D. Qu, M. Thacker, P. Castelucci, M. Bagyanszki, M.L. Epstein, J.B. Furness, Immunohistochemical analysis of neuron types in the mouse small intestine, Cell Tissue Res. 334 (2008) 147-161.

[14] T.A. Heanue, V. Pachnis, Enteric nervous system development and Hirschsprung's disease: advances in genetic and stem cell studies, Nat. Rev. Neurosci. 8 (2007) 466-479.

[15] K.M. Sanders, T. Ordog, S.D. Koh, S. Torihashi, S.M. Ward, Development and plasticity of interstitial cells of Cajal, Neurogastroenterol. Motil. 11 (1999) 311-338. 